\title{
Connection between liquid and non-crystalline solid phases in water
}

Fausto Martelli, ${ }^{1,2, a)}$ Fabio Leoni, ${ }^{2,3}$ Francesco Sciortino, ${ }^{3}$ and John Russo ${ }^{2,3}$

1) IBM Research Europe, Hartree Centre, Daresbury WA4 4AD, United Kingdom

${ }^{2)}$ School of Mathematics, University of Bristol, Bristol BS8 1UG, United Kingdom

${ }^{3)}$ Department of Physics, Sapienza University of Rome, P.le Aldo Moro 5, 00185 Rome, Italy

The origin of water anomalies hides in an experimentally inaccessible region of the phase diagram known as no-man's land, bounded at low temperature by the domain of stability of amorphous glasses, and at high temperature by the homogeneous nucleation line, below which liquid water looses its metastability. The existence of at least two different forms of glass on one side, i.e. the low-density amorphous (LDA) and the high-density amorphous (HDA) ices, and of one anomalous liquid on the other side, points to a hidden connection between these states, whose understanding has the potential to uncover what happens in no-man's land and shed light on the complex nature of water's behaviour. Here we develop a Neural Network scheme capable of discerning local structure beyond tetrahedrality. Applied over a wide region of the water's phase diagram, we show that the local structures that characterize both LDA and HDA amorphous phases are indeed embedded in the supercooled liquid phase. Remarkably, the rapid increase in the LDA-like population with supercooling occurs in the same temperature and pressure region where thermodynamic fluctuations are maximized, linking these structures with water's anomalies. At the same time, the population of HDAlike environments rapidly increases with pressure, becoming the majority component at high density. Our results show that both LDA and HDA are genuine glasses, and provide a microscopic connection between the non-equilibrium and equilibrium phase diagrams of water.

\section{INTRODUCTION}

Water is unique in many ways. Its crystalline phase diagram is by far the most complex of any pure substance, and still far from being fully explored [1-3]. But even more surprising is the existence of multiple amorphous ice forms. The first one is the low-density amorphous ice (LDA), likely the most abundant form of ice in the universe, that can be obtained, for example, by rapid quench of liquid water from ambient conditions to very low temperatures. By compressing isothermically LDA or hexagonal ice I $h$, a first-order-like phase transition is encountered which produces high-density amorphous ice (HDA) with a density increase in the order of $\sim 20-25 \%$ [4]. Remarkably, LDA can be recovered by isothermal decompression and isobaric heating of HDA at different pressures [5].

Broadly defined, the LDA and HDA acronym encompass a larger set of sub-families characterized by different thermal histories [6-11] such as the LDA-I and LDAII [7], a more ordered low-density phase obtained upon heating ice VIII [12], the annealed and very high-density amorphous ices, eHDA [6] and vHDA [13, 14]. Furthermore, intriguing connections worth further investigations between HDA (produced upon isothermal compression of $\mathrm{I} h$ or of LDA) and ice IV have been recently reported [8, $10]$.

The very nature of amorphous ices is therefore still highly debated, but even less is known about their connection to the liquid state [15]. The liquid phase is hypothesized (with support from numerical simulations)

a)Electronic mail: fausto.martelli@ibm.com to exist in two different phases below its second critical point, named low-density liquid (LDL) and high-density liquid (HDL) respectively [16-23].

The possibility of a continuous thermodynamic link between LDA and HDA, and the supercooled liquids (LDL and HDL) is highly debated. Most experimental works have focused on exploring the glass-to-liquid transition upon heating of the amorphous ices, either via calorimetry or dielectric relaxation spectroscopy. Two distinct glass transitions have been considered for LDA [2426] and HDA [27-32] respectively, but questions remain whether the weak calorimetric signatures can instead be interpreted as an orientational glass transition for LDA [33-35], and whether HDA is a mechanically collapsed state with no connection to the supercooled liquid $[1,8,36]$.

In this article we bypass the difficulties associated with characterizing a glass (out-of-equilibrium) transition, and instead explore the structural similarity between the different disordered configurations in their regions of metastability. Probing the connection between amorphous states is a challenging task, mostly because of the lack of theoretical and/or experimental tools able to account for the high degeneracy of local configurations in statistically isotropic materials [37]. In particular, previous studies made stringent assumptions of a specific local structure, starting from the metastable liquid state, and extrapolating towards the glass state. In this study we instead make no assumptions on the local structure, encompassing all medium-ranged configurations, and start from the glass state, probing its structural similarity with the liquid state. To this end, we adopt two complementary strategies. The first one consists in mapping the structural properties of the local environment surrounding a water molecule onto a high dimensional function 
(or order parameter) of the particle positions, that allows us to effectively characterize and distinguish liquid, amorphous, and glass phases. The second key step is to classify each local environment using Neural Networks (NN) that have been proven to be a flexible and powerful tool to deal with high-dimensional order parameters, and are recently finding wide applications in water [3841], and to a lesser extent to out-of-equilibrium systems such as glasses [42-44].

Using NN allows us to automatically classify molecules in different categories (or outputs, that are LDA, HDA, and the high- $T$ liquid phase) based on their local environments, as probed by a multidimensional set of bondorientational order parameters [45] (BOOs). After training the NN with configurations of water in the LDA, HDA, and high- $T$ liquid phases (at thermodynamic conditions specified in Fig. 1), we apply it to equilibrated supercooled liquid configurations at three different pressures $\left(10^{-4}, 0.04\right.$, and $\left.0.1 \mathrm{GPa}\right)$ and a wide range of temperatures $(190<T<250 \mathrm{~K})$ to quantify the possible presence of local environments which symmetries are recognized by the $\mathrm{NN}$ as the ones present in LDA and HDA amorphous glasses. We stress that our HDA training set includes configurations of HDA down to ambient pressure, obtained by decompression of the highpressure HDA samples. This ensures that our training set for each amorphous ice spans all the pressure range of our metastable liquid configurations. The inclusion of high- $T$ liquid configurations in the training set is used to give the NN the ability to identify disordered hydrogenbond network configurations, and allows us to check the high- $T$ consistency of our analysis.

In Fig. 1a we reproduce the phase diagram of the TIP4P/2005 water model [46], here adopted to describe water molecules. The orange squares and circles represent the loci of specific heat $\left(c_{P}\right)$ and isothermal compressibility $\left(k_{T}\right)$ maxima respectively, and are plotted in Fig. 1c, for pressures $P=10^{-4}, 0.04,0.1 \mathrm{GPa}$. These maximum lines converge towards the liquid-liquid critical point (LLCP, star symbol in fig. 1a) recently reported in Ref. [23]. In Fig. 1a, the LDA state points are represented as red symbols, while HDA state points are represented as green symbols. A first order-like phase transition is signalled by the density hysteresis cycle obtained from the isothermal compression/decompression cycle and shown in Fig. 1b for $T=80,100,120,140 \mathrm{~K}$. The transition point in correspondence with the sudden increase in the density is taken as the point in which $\left(\partial^{2} \rho / \partial P^{2}\right)_{T}=0$, and is plotted with a continuous black line in Fig. 1a. This transition line tends towards the LLCP with increasing $T$. This connection between an out-of-equilibrium transition (the pressureinduced LDA-to-HDA transition) and the metastable equilibrium LLCP, suggests a close correspondence between the amorphous ices and the corresponding liquids. We explore it below.
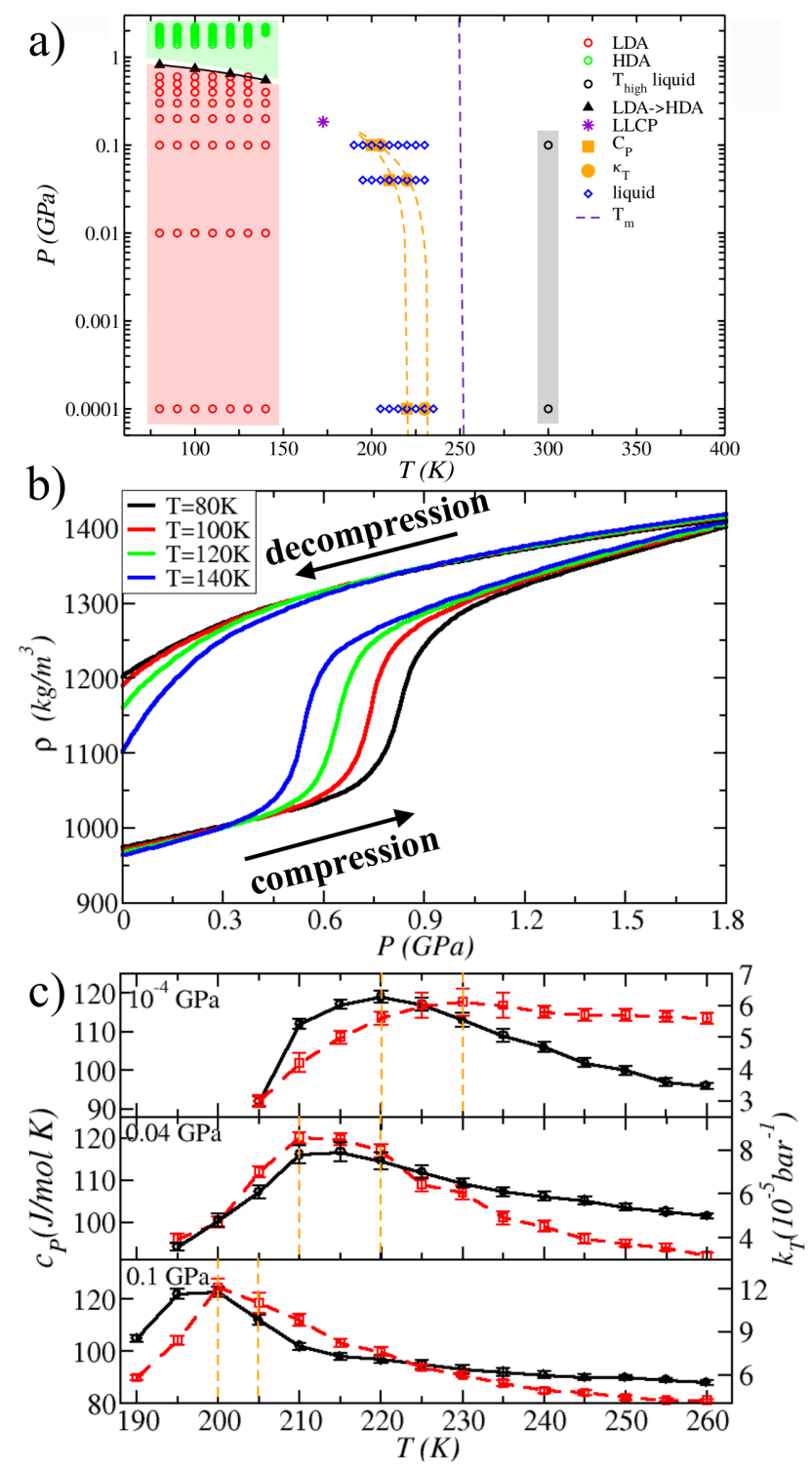

FIG. 1. (a) Phase diagram of the TIP4P/2005 model. (b) Density profile during the isothermal compression/decompression cycle of LDA to HDA at four temperatures indicated in the legend. The sudden increase in the density moves at lower pressure increasing the temperature. (c) Specific heat $\left(c_{P}\right.$, black circles) and isothermal compressibility ( $k_{T}$, red squares) as a function of temperature for the three different pressures (from top to bottom) $P=10^{-4}, 0.04,0.1 \mathrm{GPa}$. The dashed orange vertical lines represent the loci of maxima in $c_{P}$ and $k_{T}$.

\section{METHODS}

In this Section we describe the protocol we have adopted in our simulations and the Neural Network setup. We also give a brief description of the two-state liquid theory that is employed for theoretical comparison with our numerical results. 


\section{A. Equilibrium and out-of-equilibrium simulations}

Our study is based on classical molecular dynamics simulations of systems composed of $N=512, N=8192$ and $N=21952$ rigid water molecules described by the TIP4P/2005 interaction potential [46] in the isobaric $(N P T)$ ensemble. We have run classical molecular dynamics simulations with GROMACS 2018.4 [47] running on IBM POWER8 machines with NVIDIA Kepler K80 GPUs.

Equilibrated trajectories with $N=512$ water molecules have been presented in Ref. [48]. We have employed Nosé-Hoover thermostat [49, 50] with 0.2 ps relaxation time to maintain constant temperature, and Parrinello-Rahman barostat [51] with 2 ps relaxation time to maintain constant pressure. We have truncated short-range interactions at $9.5 \AA$, and we have computed long range electrostatic terms using particle mesh Ewald with a grid spacing of $1.2 \AA$. At each state point, we have computed and carefully monitored the decay of the selfpart of the intermediate scattering function (ISF) with time [52]. All reported trajectories are at least 500 times longer that the structural relaxation time as computed from the ISF. Depending on the thermodynamic conditions, production runs vary between $50 \mathrm{~ns}$ and $12 \mu \mathrm{s}$. No sign of crystallization have been observed. For each state point we have run 5 independent trajectories.

Out-of-equilibrium simulations treat samples of $N=$ 8192 and $N=21952$ water molecules arranged in rectangular parallelepiped boxes. Coulombic and LennardJones interactions are calculated with a cut off distance of $1.1 \mathrm{~nm}$ and long-range electrostatic interactions are treated using the Particle-Mesh Ewald algorithm. Temperatures and pressures are controlled using a NoséHoover thermostat $[49,50]$ and a Berendsen barostat [53]. For the thermostat, the period of the kinetic energy oscillations between the system and the reservoir is set to $1 \mathrm{ps}$, while the time constant for the barostat is set to 1 ps. Following Ref. [10], we have produced LDA simulating the quenching of equilibrated liquid water at $T=300 \mathrm{~K}$ to $T=80 \mathrm{~K}$ at a cooling rate of $1 \mathrm{~K} / \mathrm{ns}$. We have produced HDA simulating the isothermal compression of LDA and of $\mathrm{I} h$ samples at four temperatures, namely $T=80 \mathrm{~K}, T=100 \mathrm{~K}, T=120 \mathrm{~K}$ and $T=140 \mathrm{~K}$ at a compression rate of $0.01 \mathrm{GPa} / \mathrm{ns}$ reaching up to $3.0 \mathrm{GPa}$. We have then simulated the isothermal decompression of HDA from 2.0 GPa to $10^{-4} \mathrm{GPa}$ at a decompression rate of $0.01 \mathrm{GPa} / \mathrm{ns}$. Each simulation here presented is the average over 10 independent simulations.

\section{B. The Neural Network (NN)}

Here we employ supervised learning because, at the thermodynamic conditions of interest in the present work, we hypothetically know all possible phases, which are the output of the NN, excluding for example the formation of crystalline phases (see Refs.[10, 48]). We use a

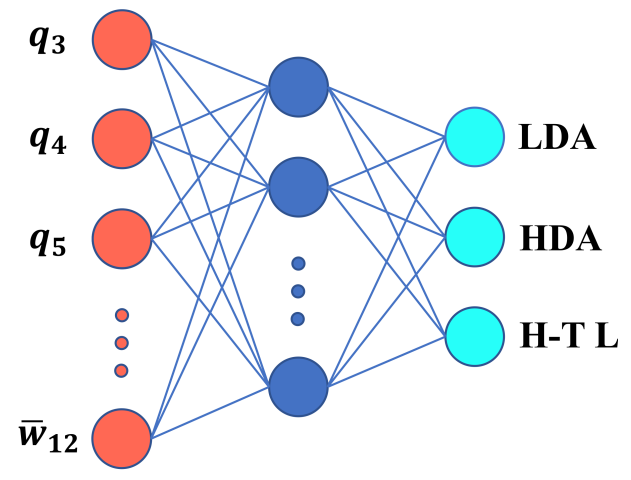

FIG. 2. Schematic representation of the Neural Network designed for the present study. Each of the 30 nodes of the input layer consists of one of the structural bond orientational order parameter defined in eqs. 1 and 3. The hidden layer has 10 nodes, while the output has 3 nodes for the LDA, the HDA and the high- $T$ liquid phases respectively.

single layer feed-forward NN composed of an input layer (IL), an hidden layer (HL) and an output layer (OL). The input signal associated to the position of each oxygen atom is embedded in order parameters represented by a 30-dimensional vector (the IL has 30 nodes, see below). The NN transforms each input signal into an output represented by LDA, HDA, and the high- $T$ liquid phase (the OL has 3 nodes). We set the number of nodes of the HL to 10 . In fig. 2 we report a schematic representation of the Neural Network setup. We initialize the weights following the Xavier method [54], consisting on setting random weights from a normal distribution with zero mean and variance equal to 2 divided by the sum of the number of nodes in the IL and the OL. We consider the sigmoid, or logistic function, as activation function for both IL-HL and HL-OL. As error function we chose the overall mean square error between the actual and target outputs. To optimize the weights of the network we minimize the error function using the stochastic gradient descent and backpropagation methods. We set the learning rate to $\alpha=0.01$ and the number of epochs to 90. The performance of the NN is above $98 \%$ for a wide range of learning rates and epochs, indicating that the network is robust. To rule out overfitting we verified to get the same performance from both test and training sets.

The input vector describing the local environment of each oxygen atom is composed of 30 different local OPs, first introduced by Steinhardt et. al. [45]. These OPs are based on the complex vector $q_{l m}(i)$ and its average $\bar{q}_{l m}(i)$

$$
\begin{gathered}
q_{l m}(i)=\frac{1}{N_{b}(i)} \sum_{j=1}^{N_{b}(i)} Y_{l m}\left(r_{i j}\right), \\
\bar{q}_{l m}(i)=\frac{1}{N_{b}(i)+1} \sum_{k \in\left\{i, N_{b}(i)\right\}} q_{l m}(k),
\end{gathered}
$$

where $N_{b}(i)$ is the number of neighbors of particle $i$ 
$\left(N_{b}=16\right.$ in this work), $l$ and $m$ are integers with $m \in[-l, l], Y_{l m}\left(r_{i j}\right)$ are the spherical harmonics, and $r_{i j}$ is the position vector from particle $i$ to $j$. The sum in the definition of $\bar{q}_{l m}(i)$ runs over the $N_{b}(i)$ neighbors plus the particle $i$ itself. The set of $l$ spherical harmonics defines an orthonormal basis spanning the $(2 l+1)$-dimensional representation of the rotation group $\mathrm{SO}(3)$ relating the irreducible representation of $\mathrm{SO}(3)$ and the symmetries of crystalline structures. The average spherical harmonics depend on the choice of the reference frame, but their average over $m$ are rotationally invariant and encode an intrinsic property of the medium. The rotationally invariant $q_{l}(i)$ and their averages $\bar{q}_{l}(i)$ are therefore defined as

$$
\begin{aligned}
& q_{l}(i)=\sqrt{\frac{4 \pi}{2 l+1} \sum_{m=-l}^{l}\left|q_{l m}(i)\right|^{2}}, \\
& \bar{q}_{l}(i)=\sqrt{\frac{4 \pi}{2 l+1} \sum_{m=-l}^{l}\left|\bar{q}_{l m}(i)\right|^{2}} .
\end{aligned}
$$

The cubic OPs $w_{l}(i)$ and their average $\bar{w}_{l}(i)$ are defined as

$$
\begin{gathered}
\sum_{m_{1}+m_{2}+m_{3}=0}\left(\begin{array}{ccc}
l & l & l \\
m_{1} & m_{2} & m_{3}
\end{array}\right) q_{l m_{1}}(i) q_{l m_{2}}(i) q_{l m_{3}}(i) \\
\left(\sum_{m=-l}^{l}\left|q_{l m}(i)\right|^{2}\right)^{3 / 2} \\
\sum_{\frac{m_{1}+m_{2}+m_{3}=0}{}\left(\begin{array}{ccc}
l & l & l \\
m_{1} & m_{2} & m_{3}
\end{array}\right) \bar{q}_{l m_{1}}(i) \bar{q}_{l m_{2}}(i) \bar{q}_{l m_{3}}(i)}^{\left(\sum_{m=-l}^{l}\left|\bar{q}_{l m}(i)\right|^{2}\right)^{3 / 2}}
\end{gathered}
$$

where the term in parentheses is the Wigner $3 j$ symbol. The 30-dimensional vector used as input for the NN is composed by the following combination of BOOs: $q_{l}(i)$ and $\bar{q}_{l}(i)$ with $l \in[3,12]$, and $w_{l}(i)$ and $\bar{w}_{l}(i)$ with $l$ even and $l \in[4,12]$. For higher values of $l$ the average period of the oscillations of the Legendre polynomials in the cosspace becomes smaller than the scattering of some characteristic angle, and the corresponding invariants rapidly vanish. We therefore do not consider values of $l$ larger than 12.

We trained the NN with 2000 configurations of each of the target phases. The configurations have been selected from a wide range of values in the existence domain of each phase reported in Fig. 1a.

\section{Two-state model}

Two-state models of water describe the anomalies of water as the structural change that occurs locally to the network of hydrogen-bonded molecules [55-61]. The local environments surrounding each water molecule are divided in two populations which behave differently when the thermodynamic conditions change. An ordered population includes all environments that are energetically favoured, which have a high degree of local order and a high specific volume, and are thus the (free-energy) preferred state at low-temperatures and low-pressures. The other population instead includes all environments that are entropically favoured, with a high degeneracy and a low specific volume, and which instead are the majority component at high temperatures and high-pressures. Evidence for this bimodality has been corroborated by analysis of numerical data [61-63]. The two states are easily distinguished by their temperature/pressure dependence. The fraction of ordered states, which we indicate with the symbol $s$, increases with decreasing temperature and pressure, while the fraction of disordered states $(1-s)$ increases in the opposite direction.

Two-state models have been very successful in accurately describing the equation of state of water, and also include the possibility of a phase separation of the two states in different thermodynamic phases: the LDL phase would have ordered states as the majority component $(s>0.5)$ and HDL phase would have a majority component of disordered states $(s<0.5)$. The two-state model thus considers water as a regular mixture, with the difference that the composition of the mixture is not fixed but changes depending on the thermodynamic conditions. For a broader introduction to two-state models and for the free-energy and anomalies expressions we refer to Ref. [62].

In the following we will interpret the Neural Network classification scheme results in terms of the two-state description. To facilitate a comparison we have thus derived new two-state model parameters for TIP4P/2005. We consider simulations of 512 water molecules spanning thermodynamic conditions in the liquid state ranging from $T=200 \mathrm{~K}$ to $T=350 \mathrm{~K}$, and pressures from $P=-1$ kbar to $P=3$ kbar. For each state point, the fraction of locally favored structures is then extracted with the $\zeta$ order parameter [62], which distinguishes order structures from the distance distribution between the first and second shell of nearest neighbors. From the $(T, P)$ dependence of $s$ we fit the two-state model equation of state [62] whose fitting parameters are the coefficients of a second order expansion of the bulk free energy difference between the two states around the critical point

$$
\Delta G=a_{1} \hat{T}+a_{2} \hat{P}+a_{11} \hat{T}^{2}+a_{12} \hat{T} \hat{P}+a_{22} \hat{P}^{2}
$$

where $\hat{T}=\left(T-T_{c}\right) / T_{c}$ and $\hat{P}=\left(P-P_{c}\right) / P_{c}$, and the critical temperature $T_{c}=172 \mathrm{~K}$ and critical pressure $P_{c}=0.1861 \mathrm{GPa}$ are taken from a detailed study of critical fluctuations in the TIP4P/2005 model [23]. The resulting two-state model is determined by the coefficients (expressed here in unit of temperature) $a_{1} / k_{B}=306 \mathrm{~K}$, $a_{2} / k_{B}=195 \mathrm{~K}, a_{11} / k_{B}=399 \mathrm{~K}, a_{12} / k_{B}=60.3 \mathrm{~K}$, $a_{22} / k_{B}=44.3 \mathrm{~K}$, where $k_{B}$ is the Boltzmann constant. 


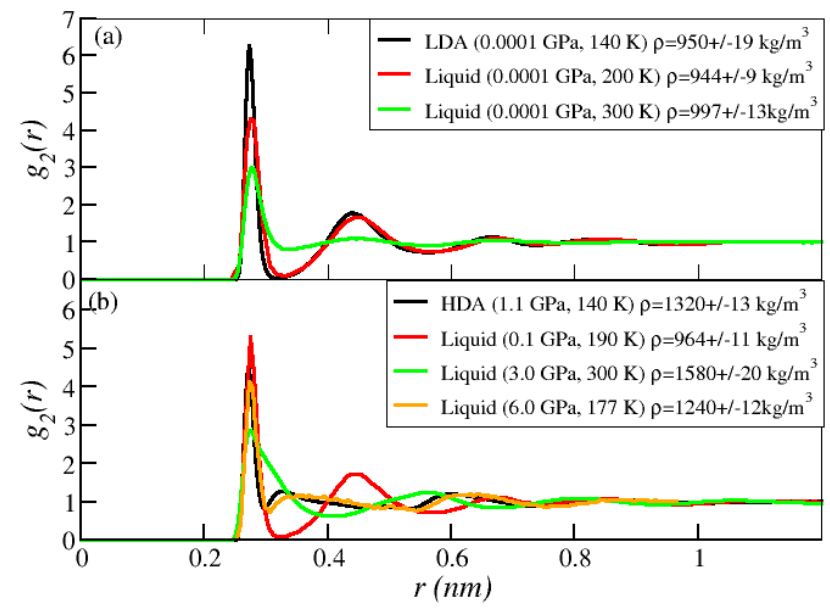

FIG. 3. Two-bodies pair correlation function $g_{2}(r)$ for liquids and amorphous states at different thermodynamic conditions. Panel (a): $g_{2}(r)$ for LDA at $P=0.0001 \mathrm{GPa}$ and $T=140 \mathrm{~K}$ (black), liquid water at $P=0.0001 \mathrm{GPa}$ and $T=200 \mathrm{~K}$ (red) and liquid water at $P=0.0001 \mathrm{GPa}$ and $T=300 \mathrm{~K}$ (green). Panel (b): $g_{2}(r)$ for HDA at $P=1.1 \mathrm{GPa}$ and $T=$ $140 \mathrm{~K}$ (black), liquid water at $P=0.1 \mathrm{GPa}$ and $T=190 \mathrm{~K}$ (red), liquid water at $P=3.0 \mathrm{GPa}$ and $T=300 \mathrm{~K}$ (green), and liquid water at $P=6.0 \mathrm{GPa}$ ant $T=177 \mathrm{~K}$ (orange). Individual densities are reported in the legend.

\section{RESULTS}

In this section we present and discuss the main findings of this work.

\section{A. Two-body pair correlation functions}

We start our investigation by inspecting the two-body pair correlation function, $g_{2}(r)$, that describes how the density of particles varies as a function of the distance from a tagged particle. In Fig. 3 we show the oxygenoxygen $g_{2}(r)$ for both amorphous ices and the liquid phase. Panel (a) compares LDA at $T=140 \mathrm{~K}$ (black) with the liquid at $T=200 \mathrm{~K}$ (red), and the liquid at $T=300 \mathrm{~K}$ (green), at ambient pressure. Upon cooling the liquid phase from $T=300 \mathrm{~K}$ to $T=200 \mathrm{~K}$, we observe a progressive depletion of the population in the interstitial region between the first and the second peak, with a corresponding structurization of the first and the second peak, indicative of an enhancement of the tetrahedrality of the liquid structure upon cooling. Further structurization occurs in LDA, as indicated by the intensification of the first peak. We observe that the peak positions of the liquids are remarkably consistent with the peak positions of LDA, and that the distribution of LDA and liquid at $T=200 \mathrm{~K}$ qualitatively overlap, suggesting some intimate structural correlation between the liquid phase and LDA. In panel (b) of Fig. 3 we can see that HDA is characterized by unique features such as a minimum at $\sim 0.3 \mathrm{~nm}$ followed by an asymmetric second peak spreading from $\sim 0.33$ to $\sim 0.53 \mathrm{~nm}$, and a third peak located at $\sim 0.6 \mathrm{~nm}$. These features are found in the metastable liquid phase only at considerably higher pressures but at comparable densities. To show this we compare the pair correlation function at three different state points, outside the conditions of stability of the HDA phase. The first point, $T=190 \mathrm{~K}$ and $P=0.1 \mathrm{GPa}$ (low $T$ - low $P$ ), shows a distribution drastically different from HDA, with deep minima between the peaks. The second point, $P=3.0 \mathrm{GPa}$ and $T=300 \mathrm{~K}$ (high $T$ - high $P)$, is characterized by a distribution with a low, broad first peak and a second peak located at $\sim 0.56 \mathrm{~nm}$, not far from the third peak in HDA. Finally, at $P=6.0 \mathrm{GPa}$ and $T=177 \mathrm{~K}$ (low $T$ - high $P$ ), where the HDA and liquid phase have comparable densities $\left(\sim 1300 \mathrm{~kg} / \mathrm{m}^{3}\right)$, we observe a good correspondence between the pair correlation functions. These results suggest that the thermodynamic connection between HDA and the liquid phase may not be straightforward. To further shed light on structural similarities between HDA and liquid water we extend our investigation by performing a multipoint (beyond the pair level) analysis of the structural properties of all phases here considered.

\section{B. Populations in the supercooled phase}

To map the local environments of each molecule on a high-dimensional order parameter we use the space of bond-orientational order parameters (BOOs) $q_{l}$ and $w_{l}$, which are rotationally invariant quantities obtained from combination of spherical harmonics of order $l$ [45]. Depending on the value of $l$, BOOs capture different symmetries eventually present in the local environment that we define as the set of the closest 16 neighbours in order to investigate medium-range order (beyond nearestneighbours) where structural similarities between the liquid and the amorphous ices are not granted. Our combination of BOOs includes all values of $l$ from 3 to 12 , and defines an order parameter that projects the local structural properties around each water molecule onto a 30-dimensional space. We then partition this space by training a NN, to differentiate the amorphous ices environments, HDA and LDA, and the high-T liquid. In Fig. 1a we plot as red and green symbols the state points which we have used to train the network against LDA and HDA respectively, while further configurations of HDA obtained upon isothermal decompression of HDA from $P=2.0 \mathrm{GPa}$ to ambient pressure used to train the network are not shown in the figure. In the NN training set, we also include a high-temperature (above melting) liquid environment, which represents local environments with positional correlations typical of simple liquids (black region in the figure). In summary, the NN takes the 30dimensional representation of a local environment and maps it into one of the following phases: LDA, HDA, and high- $T$ liquid. After confirming that the $\mathrm{NN}$ has a misclassification ratio below $2 \%$ between these phases, we 

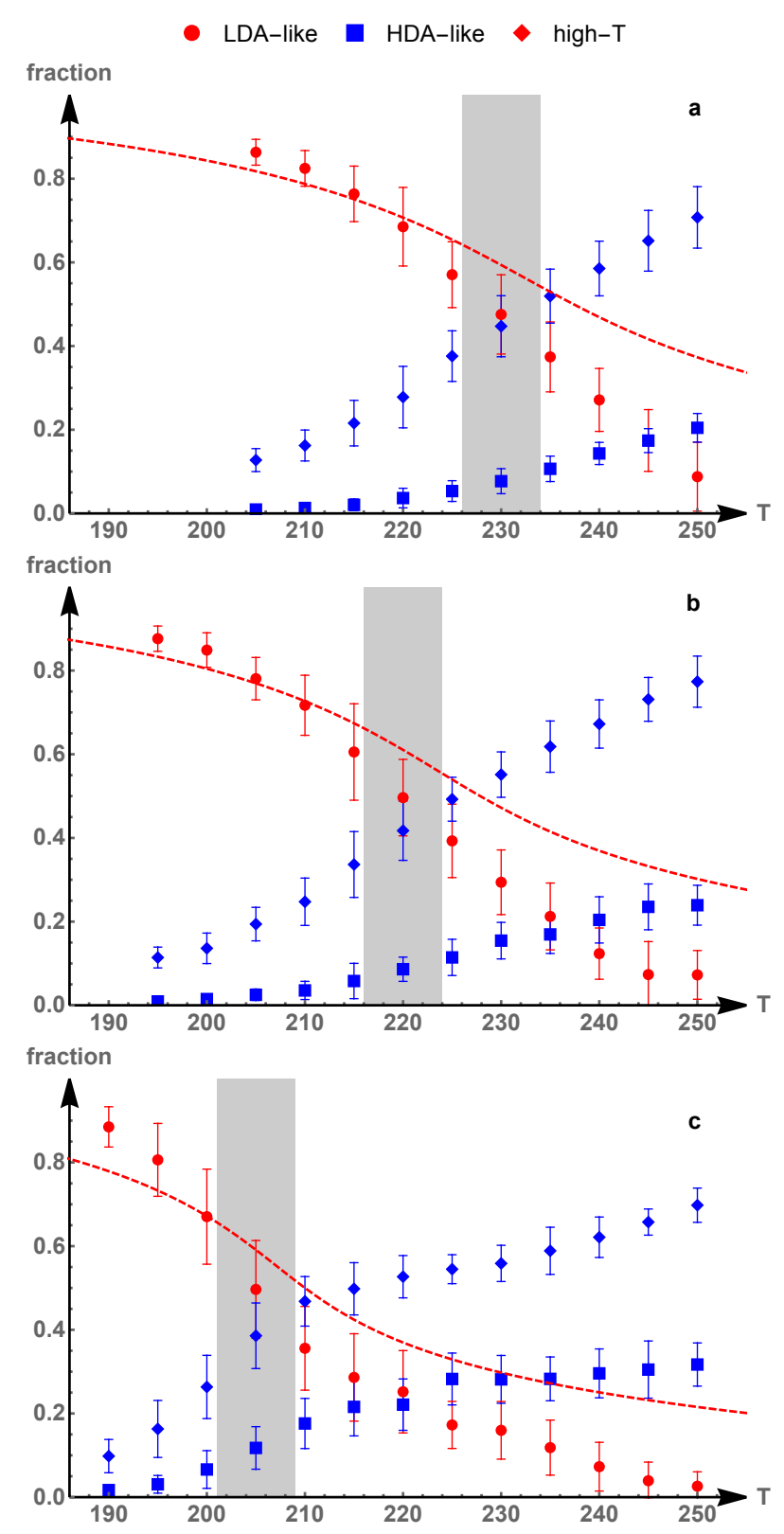

FIG. 4. Fraction of local environments as a function of the temperature for equilibrated liquid water at three pressures, (a) $P=10^{-4} \mathrm{GPa}$, (b) $P=0.04 \mathrm{GPa}$, and (c) $P=0.1 \mathrm{GPa}$. Red circles, blue squares, and blue diamonds represent the fraction of LDA-like, HDA-like, and high- $T$ liquid-like environments respectively. The dashed line represents the fraction of locally favored structures according to the two-state model for the $\zeta$ parameter. The gray band shows the location of the $k_{T}$ anomaly.

apply the order parameter to supercooled water configurations, to see whether signatures of amorphous states are present in equilibrium liquid configurations.

Our equilibrated liquid samples cross the lines of maxima of $c_{p}$ and $k_{T}$ (leftmost and rightmost vertical dashed lines in Fig. 1, respectively [48]) at three pressures, namely $P=10^{-4}, P=0.04$ and $P=0.1 \mathrm{GPa}$, and explore temperature ranges from ambient to deeply supercooled conditions. These configurations are plotted as blue points in Fig. 1a. We then apply the order parameter on the equilibrated liquid configurations and compute the fraction of each target state using the NN previously trained.

The composition of the different populations is reported in Fig. 4 for the three pressures here considered. Symbols represent the population of LDA-like (circles), HDA-like (squares), and high- $T$ (diamonds) local environments. We first note that the curves are continuous, with relatively small error bars (result which is not expected a priori from the NN classification method) and they behave as expected in the limiting cases: with the majority component being the LDA-like and high- $T$ environments at low and high temperature respectively, and with a fraction of HDL-like environments increasing with pressure. The populations can be divided in two-groups depending on whether their composition increases (HDL-like and high- $T$ ) or decreases (LDA-like) with temperature. These groups can be associated with the two states that are invoked in the explanation of water anomalies. The ordered state comprises LDA-like environments, while HDL-like and high- $T$ environments make up the disordered state. We further observe that the point where the fraction of LDA-like populations is equal to $1 / 2$ (Schottky line) falls within the region of maximum structural fluctuations for all pressure considered. In Fig. 4 this is represented as the region of compressibility $\left(k_{T}\right)$ maximum as the grey vertical bands. This result is in agreement with two-state theories of supercooled liquid water [56-61], as the region of maximum anomaly occurs where the derivative of $s$ with respect to temperature is an extremum. For comparison, the temperature dependence of fraction of the ordered state $(s(T))$ of a two-state model for a different order parameter $(\zeta$, see Methods) is presented as the red dashed lines in Fig. 4. Also this model shows that the inflection point of the $s(T)$ falls within the region of maximum anomaly. Note that the two-state model (red dashed line) and the LDA-like population (red circles), do not necessarily superimpose as they are obtained from different order parameters $(\zeta$ for the two-state model, and $\mathrm{BOO}$ for the LDA-like populations): what is physically relevant is the location of the flex point in the two curves.

Without making any assumptions on the nature of the ordered state, we have shown that it overlaps largely with the population of LDA-like local environments. This already points to a direct connection between LDA and the supercooled liquid state from a structural point of view beyond the two-particle level that was noted in the radial distribution functions (Fig. 3).

We now focus on the remaining populations, the HDAlike and high- $T$ local environments. In the language of two-state models, these environments are often modeled as a high-density disordered state [56, 64], which is a state stabilized by entropy at high-temperature, and by its high density (compared to the ordered state) at high- 


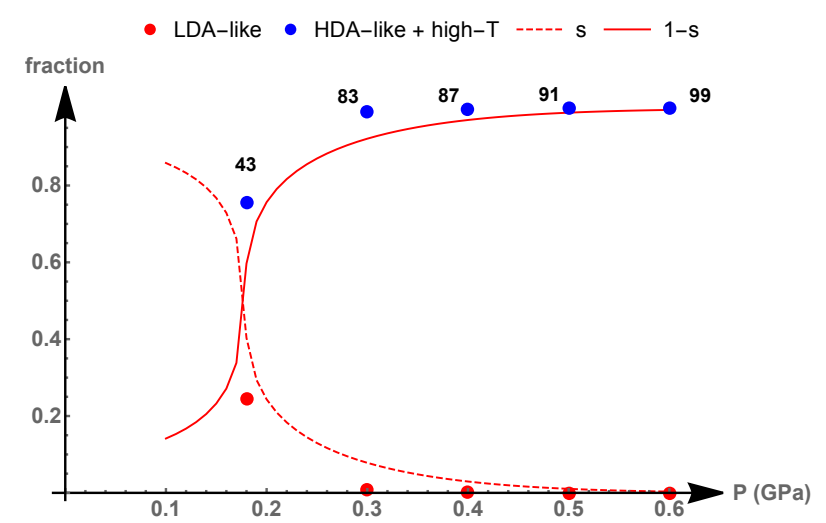

FIG. 5. Fraction of local environments as a function of the pressure for equilibrated liquid water at $\mathrm{T}=177 \mathrm{~K}$. Blue dots represent the fraction of HDA and high- $T$ liquid-like environments, red circles the fraction of LDA-like environments. The labels on blue symbols represent the relative fraction of HDAlike environments in the HDA+high- $T$ state (e.g. the label 43 indicates a relative composition of $43 \%$ HDA-like environments and $57 \%$ high- $T$ liquid-like environments). The dashed line represents the fraction $(s)$ of locally favoured structures according to the two-state model for the $\zeta$ parameter, while the continuous line is the fraction $(1-s)$ of disordered states.

pressure. These two different stabilization factors are reflected in the populations of HDA-like and high- $T$ local environments in Fig. 4. In fact, we observe that the relative composition of the disordered state changes with thermodynamic conditions. As expected, the fraction of high- $T$ structures increases with temperature, but the relative amount of HDA-like structures over high- $T$ structures increases with pressure (panels from top to bottom in Fig. 4).

To fully uncover the role of HDA-like structures, we perform additional simulations at low temperature $(T=$ $177 \mathrm{~K}$ ) and high pressures (ranging from $P=0.18$ to $0.6 \mathrm{GPa}$ ). We limit the system size to $N=300$ due to the difficulty of equilibrating liquid configurations at those extreme conditions. We then perform the $\mathrm{NN}$ analysis and report the following compositions (see Fig. 5). We observe that the fraction of HDA-like environments increases from $43 \%$ at $P=0.18 \mathrm{GPa}$, to $99 \%$ at $P=0.6 \mathrm{GPa}$. This result shows that HDA-like structures dominate the disordered state at high pressures, thus establishing a clear structural link between the HDA glassy phase and the high-pressure metastable liquid.

The behaviour extracted from the NN is consistent between different thermodynamic conditions and points to a direct link between water glasses and structures present in the supercooled liquid state. To test the classification consistency of our approach in the following section we will investigate the behaviour of the system close to its second critical point.

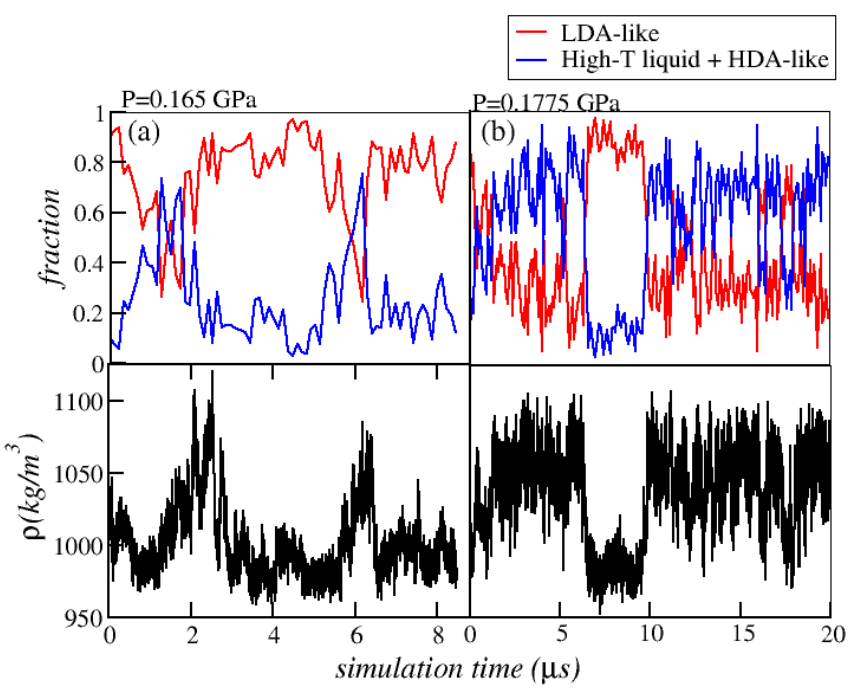

FIG. 6. Fraction of local environments and corresponding critical density fluctuations for a sample of $N=300$ water molecules in the proximity of the second critical point for the TIP4P $/ 2005$ water model at $T=177 \mathrm{~K}$. Panel (a) reports the fraction of LDA-like (red) and the sum of high$T$ and HDA-like (blue) local environments at a pressure of $0.165 \mathrm{Gpa}$. Panel (b) reports the same fractions at a pressure of $0.1775 \mathrm{GPa}$. The corresponding density fluctuations are reported in the lower panels.

\section{Populations approaching the second critical point}

It has been recently ascertained that the TIP4P/2005 model of water (along with the TIP4P/Ice model) has a liquid-liquid critical point at $T_{c}=172 \pm 1 \mathrm{~K}$ and $P_{c}=0.1861 \pm 9 \times 10^{-4} \mathrm{GPa}$ and consistent with the 3D Ising universality class [23]. In order to further test the robustness of our network and the accuracy of the order parameter, we have analyzed trajectories from Ref. [23] in the vicinity of the second critical point. In fig. 6 we report the fractional composition of a sample of $N=300$ water molecules at $T=177 \mathrm{~K}$ and $P=0.165 \mathrm{GPa}$-panel (a) - and $P=0.1775 \mathrm{GPa}$ - panel (b)- respectively. The corresponding critical density fluctuations are reported in the lower panels. It is possible to appreciate that the network is able to rationalize the contribution of LDAlike environments (red) that dominate at lower densities, and of HDA-like with high-T-like (blue) environments that dominate at higher densities. Therefore, our results indicate that our setup (the order parameter and the network) is well suited to discern among local environments also in the vicinity of criticality.

\section{CONCLUSIONS}

In conclusion, our results shed new light on the nature of the non-equilibium glassy states of water, and their connection to the metastable supercooled liquid 
state. Bypassing the difficulties associated with exploring state points near the glass transition, we establish a new method for finding direct structural links between different regions of the phase diagram. We find that both the LDA and HDA amorphous phases are genuine glassy states, which have an equilibrium counterpart at the thermodynamic conditions accessible to computer simulations. LDA-like structures are directly associated with ordered locally favoured structures, and their increase with supercooling is found to be in excellent agreement with the liquid anomalies. The disordered state is composed of both HDA-like and high- $T$ structures, the former ones found at high $P$ and low $T$, the latter at high- $T$ and low- $P$.

The generality of our approach makes it amenable for applications to other disordered condensed matter systems.

The data that support the findings of this study are available from the corresponding author upon reasonable request.

Acknowledgments. We thank Pablo Debenedetti and Gul Zerze for sharing the data published in Ref. [23]. F.M. acknowledges support from the STFC Hartree Centre's Innovation Return on Research Programme, funded by the Department for Business, Energy and Industrial Strategy. F.M. acknowledges support from the Benjamin Meaker Visiting Professorship scheme of the Institute for Advanced Studies, University of Bristol. J.R. and F.L. acknowledge support from the European Research Council Grant DLV-759187. J.R. acknowledges support from the Royal Society University Research Fellowship. F.S. acknowledges support from MIUR-PRIN 2017 Grant No. 2017Z55KCW.

\section{Correspondence.}

quests for materials should be addressed to fausto.martelli@ibm.com.

\section{References}

[1] C. G. Salzmann. "Advances in the experimental exploration of water's phase diagram". In: J. Chem. Phys. 150.6 (2019), p. 060901.

[2] M. Fitzner et al. "Ice is born in low-mobility regions of supercooled liquid water". In: Proc. Natl. Acad. Sci. 116.6 (2019), pp. 2009-2014.

[3] D. R. Moberg et al. "The end of ice I". In: Proc. Natl. Acad. Sci. 116.49 (2019), pp. 24413-24419.

[4] O. Mishima, L. D. Calvert, and E. Whalley. "Melting ice" $\mathrm{I}$ at $77 \mathrm{~K}$ and $10 \mathrm{kbar}$ : A new method of making amorphous solids". In: Nature 310 (1984).

[5] K. Winkel et al. "Water polyamorphism: Reversibility and (dis)continuity". In: J. Chem. Phys. 128 (2008), p. 044510.

[6] R. J. Nelmes et al. "Annealed high-density amorphous ice under pressure". In: Nat. Phys. 2 (2006), pp. 414-418.

[7] K. Winkel et al. "Relaxation effects in low density amorphous ice: two distinct structural states observed by neutron diffraction". In: J. Chem. Phys. 130 (2009), p. 204502.
[8] J. J. Shephard et al. "Is high-density amorphous ice simply a "derailed" state along the ice I to ice IV pathway?" In: $J$. Phys. Chem. Lett. 8.7 (2017), pp. 1645-1650.

[9] F. Martelli et al. "Large-Scale Structure and Hyperuniformity of Amorphous Ices". In: Phys. Rev. Lett. 119 (2017), p. 136002.

[10] F. Martelli et al. "Searching for crystal-ice domains in amorphous ices". In: Phys. Rev. Materials 2 (2018), p. 075601.

[11] M. Formanek and F. Martelli. "Probing the Network Topology in Network-Forming Materials: the Case of Water". In: AIP Adv. 10 (2020), p. 055205.

[12] J. J. Shephard et al. "A new structural relaxation pathway of low-density amorphous ice". In: J. Chem. Phys. 144 (2016), p. 204502.

[13] T. Loerting et al. "A second distinct structural "state" of high-density amorphous ice at $77 \mathrm{~K}$ and 1 bar". In: Phys. Chem. Chem. Phys. 3 (2001), pp. 5355-5357.

[14] J. L. Finney et al. "Structure of a new dense amorphous ice". In: Phys. Rev. Lett. 89 (2002), p. 205503.

[15] P. H. Handle, T. Loerting, and F. Sciortino. "Supercooled and glassy water: Metastable liquid (s), amorphous solid (s), and a no-man's land". In: Proc. Natl. Acad. Sci. USA 114.51 (2017), pp. 13336-13344.

[16] P. H. Poole et al. "Phase behaviour of metastable water". In: Nature 360.6402 (1992), p. 324.

[17] Y. Li, J. Li, and F. Wang. "Liquid-liquid transition in supercooled water suggested by microsecond simulations". In: Proc. Natl. Acad. Sci. 110.30 (2013), pp. 12209-12212.

[18] J. C. Palmer et al. "Metastable liquid-liquid transition in a molecular model of water". In: Nature 510.7505 (2014), p. 385 .

[19] J. C. Palmer et al. "Density and bond-orientational relaxations in supercooled water". In: Mol. Phys. 114 (2016), pp. 2580-2585.

[20] Y. Ni and J. L. Skinner. "Evidence for a liquid-liquid critical point in supercooled water within the E3B3 model and a possible interpretation of the kink in the homogeneous nucleation line". In: J. Chem. Phys. 144.21 (2016), p. 214501.

[21] J. C. Palmer et al. "Advances in computational studies of the liquid-liquid transition in water and water-like models". In: Chem. Rev. 118.18 (2018), pp. 9129-9151.

[22] J. C. Palmer et al. "Comment on "The Putative LiquidLiquid Transition is a Liquid-Solid Transition in Atomistic Models of Water" [I and II: J. Chem. Phys. 135, 134503 (2011); J. Chem. Phys. 138, 214504 (2013)]". In: J. Chem. Phys. 148 (2018), p. 137101.

[23] P. G. Debenedetti, F. Sciortino, and G. H. Zerze. "Second critical point in two realistic models of water". In: Science 369 (2020), pp. 289-292.

[24] J. A. McMillan and S. C. Los. "Vitreous ice: Irreversible transformations during warm-up". In: Nature 206.4986 (1965), p. 806.

[25] I. Kohl et al. "Water Behaviour: glass transition in hyperquenched water?" In: Nature 435.7041 (2005), E1.

[26] S. Capaccioli and K. L. Ngai. "Resolving the controversy on the glass transition temperature of water?" In: J. Chem. Phys. 135.10 (2011), p. 104504.

[27] K. Amann-Winkel et al. "Water's second glass transition". In: Proc. Natl. Acad. Sci. USA 110.44 (2013), pp. 17720-17725.

[28] N. Giovambattista, H. E. Stanley, and F. Sciortino. "Relation between the high density phase and the very-high density phase of amorphous solid water". In: Phys. Rev. Lett. 94 (2005), p. 107803. 
[29] K. Amann-Winkel et al. "Colloquium: Water's controversial glass transitions". In: Rev. Mod. Phys. 88.1 (2016), p. 011002.

[30] J. N. Stern, M. Seidl-Nigsch, and T. Loerting. "Evidence for high-density liquid water between 0.1 and 0.3 GPa near 150 K". In: Proc. Natl. Acad. Sci. USA 116.19 (2019), pp. 91919196.

[31] V. Fuentes-Landete et al. "Nature of water's second glass transition elucidated by doping and isotope substitution experiments". In: Phys. Rev. X 9.1 (2019), p. 011015.

[32] L. Xu et al. "Appearance of a fractional Stokes-Einstein relation in water and a structural interpretation of its onset". In: Nat. Phys. 5 (2009), pp. 565-569.

[33] C. A. Angell. "Insights into phases of liquid water from study of its unusual glass-forming properties". In: Science 319.5863 (2008), pp. 582-587.

[34] J. J. Shephard and C. G. Salzmann. "Molecular reorientation dynamics govern the glass transitions of the amorphous ices". In: J. Phys. Chem. Lett. 7.12 (2016), pp. 2281-2285.

[35] F. Martelli et al. "A local order metric for condensed phase environments". In: Phys. Rev. B 97 (2016), p. 064105.

[36] C. A. Tulk et al. "Absence of amorphous forms when ice is compressed at low temperature". In: Nature 569.7757 (2019), p. 542 .

[37] H. Tanaka et al. "Revealing key structural features hidden in liquids and glasses". In: Nat. Rev. Phys. (2019), p. 1.

[38] P. Geiger and C. Dellago. "Neural networks for local structure detection in polymorphic systems". In: J. Chem. Phys. 139 (2013), p. 164105.

[39] L. Zhang et al. "Deep Potential Molecular Dynamics: A Scalable Model with the Accuracy of Quantum Mechanics". In: Phys. Rev. Lett. 120 (2018), p. 143001.

[40] H. Chan et al. "Machine learning coarse grained models for water". In: J. Phys: Condens. Matter 20.49 (2008), p. 494243.

[41] H.-Y. Ko et al. "Isotope Effects in Liquid Water via Deep Potential Molecular Dynamics". In: Mol. Phys. 117 (2019), pp. 3269-3281.

[42] S. S. Schoenholz et al. "Relationship between local structure and relaxation in out-of-equilibrium glassy systems". In: Proc. Natl. Acad. Sci. USA 114.2 (2017), pp. 263-267.

[43] G. S. Hartnett, E. Parker, and E. Geist. "Replica symmetry breaking in bipartite spin glasses and neural networks". In: Phys. Rev. E 98 (2018), p. 022116.

[44] E. Boattini et al. "Autonomously revealing hidden local structures in supercooled liquids". In: arXiv preprint arXiv:2003.00586 (2020).

[45] P. J. Steinhardt, D. R. Nelson, and M. Ronchetti. "Bondorientational order in liquids and glasses". In: Phys. Rev. B 28.2 (1983), pp. $784-805$.
[46] J. L. F. Abascal and C. Vega. "A general purpose model for the condensed phases of water: TIP4P/2005". In: J. Chem. Phys. 123 (2005), p. 234505.

[47] M. J. Abraham et al. "GROMACS: High performance molecular simulations through multi-level parallelism from laptops to supercomputers". In: SoftwareX 1 (2015), pp. 19-25.

[48] F. Martelli. "Unravelling the contribution of local structures to the anomalies of water: The synergistic action of several factors". In: J. Chem. Phys. 150 (2019), p. 094506.

[49] S. Nosé. "A molecular dynamics method for simulations in the canonical ensemble". In: Mol. Phys. 52 (1984), pp. 255268.

[50] W. G. Hoover. "Canonical dynamics: Equilibrium phasespace distributions". In: Phys. Rev. A 31 (1985), p. 1695.

[51] M. Parrinello and A. Rahman. "Polymorphic transitions in single crystals: A new molecular dynamics method". In: $J$. Appl. Phys. 52 (1981), p. 7182.

[52] J.-P. Hansen and I. R. McDonald. Theory of Simple Liquids. 3rd ed. Elsevier, 2006.

[53] H. J. C. Berendsen et al. "Molecular dynamics with coupling to an external bath". In: J. Chem. Phys. 81 (1984), p. 3684.

[54] X. Glorot and Y. Bengio. In: in Proceedings of the Thirteenth International Conference on Artificial Intelligence and Statistics (2010), pp. 249-256.

[55] C. T. Moynihan. "Two species/nonideal solution model for amorphous/amorphous phase transitions". In: MRS Online Proceedings Library Archive 455 (1996).

[56] H. Tanaka. "Simple physical model of liquid water". In: $J$. Chem. Phys. 112.2 (2000), pp. 799-809.

[57] M. J. Cuthbertson and P. H. Poole. "Mixturelike behavior near a liquid-liquid phase transition in simulations of supercooled water". In: Phys. Rev. Lett. 106.11 (2011), p. 115706.

[58] A. Nilsson and L. G. m. Pettersson. "Perspective on the structure of liquid water". In: Chemical Physics 389.1-3 (2011), pp. 1-34.

[59] H. Tanaka. "Bond orientational order in liquids: Towards a unified description of water-like anomalies, liquid-liquid transition, glass transition, and crystallization". In: The European Physical Journal E 35.10 (2012), p. 113.

[60] V. Holten and M. A. Anisimov. "Entropy-driven liquid-liquid separation in supercooled water". In: Scientific reports 2.1 (2012), pp. 1-7.

[61] R. Shi, J. Russo, and H. Tanaka. "Common microscopic structural origin for water's thermodynamic and dynamic anomalies". In: J. Chem. Phys. 149.22 (2018), p. 224502.

[62] J. Russo and H. Tanaka. "Understanding water's anomalies with locally favoured structures". In: Nat. Commun. 5.1 (2014), pp. 1-11.

[63] J. M. Montes de Oca, F. Sciortino, and G. A. Appignanesi. "A structural indicator for water built upon potential energy considerations". In: J. Chem. Phys. 152.24 (2020), p. 244503.

[64] V. Holten et al. "Thermodynamics of supercooled water". In: J. Chem. Phys. 136.9 (2012), p. 094507. 